\title{
Epigenetic silencing of DUSP9 induces the proliferation of human gastric cancer by activating JNK signaling
}

\author{
FANG WU ${ }^{1,2}$, TIANMIN LV ${ }^{3}$, GANG CHEN ${ }^{4}$, HUAJUN YE $^{2}$, WEI WU ${ }^{2}$, \\ GANG $\mathrm{LI}^{5}$ and FA-CHAO $\mathrm{ZHI}^{1}$
}

\begin{abstract}
${ }^{1}$ Guangdong Provincial Key Laboratory of Gastroenterology, Department of Gastroenterology, Nanfang Hospital, Southern Medical University, Guangzhou, Guangdong; ${ }^{2}$ Department of Gastroenterology, The First Affiliated Hospital of Wenzhou Medical University; ${ }^{3}$ Department of Nursing, Medical School, Huzhou Teachers College; Departments of ${ }^{4}$ Hepatobiliary Surgery and ${ }^{5}$ Chemoradiation Oncology, The First Affiliated Hospital of Wenzhou Medical University, Zhejiang, P.R. China
\end{abstract}

Received January 2, 2015; Accepted April 3, 2015

DOI: 10.3892/or.2015.3998

\begin{abstract}
Dual-specificity phosphatase 9 (DUSP9) is a strong negative regulator of transcription factor activating kinases (ERK, JNK and p38) in the mitogen-activated protein kinase (MAPK) pathways. The aim of this study was to examine the $\mathrm{CpG}$ island methylation status of DUSP9 using bisulfite sequencing PCR (BSP) in gastric cancer (GC). The investigation was conducted on 30 clinical GC samples and selected corresponding tumor-free normal gastric mucosa tissues, using BSP for the determination of the promoter methylation status. The methylation status of the tumor samples was compared to the corresponding tumor-free samples. DUSP9 was silenced by promoter region hypermethylation and $\mathrm{G} 2 / \mathrm{M}$ phase arrest was induced by DUSP9 in the MKN-1 GC cell line. MKN-1 proliferation was suppressed by DUSP9 by inhibiting c-Jun, which was induced by JNK signaling. The expression levels of CCND1, c-Jun, CDK4 and CDK6 were upregulated while p21 was downregulated by DUSP9 in MKN-1 cells. However, DUSP9-induced resulted in the regulation of the levels of cycle-related molecules, whivh were inhibited when the JNK inhibitor SP600125 was added. In conclusion, DUSP9 was frequently methylated in human GC and the expression of DUSP9 is silenced by promoter region hypermethylation.
\end{abstract}

Correspondence to: Professor Gang Li, Department of Chemoradiation Oncology, The First Affiliated Hospital of Wenzhou Medical University, 2 Fuxue Road, Wenzhou, Zhejiang 325000, P.R. China E-mail: gangliwmu@163.com

Professor Fa-Chao Zhi, Guangdong Provincial Key Laboratory of Gastroenterology, Department of Gastroenterology, Nanfang Hospital, Southern Medical University, 1838 Guangzhou Road, Guangzhou, Guangdong 510515, P.R. China

E-mail: zfcfimmu@163.com

Key words: DNA methylation, DUSP9, gastric cancer, proliferation, JNK signaling
The results of this study, combined with previous studies, suggested that therapeutic intervention to increase the expression or activity of DUSP9 may enable the activation of anti-proliferation signals in malignant cells.

\section{Introduction}

A total of 989,600 new gastric cancer (GC) cases and 738,000 mortalities are estimated to have occurred in 2008, accounting for $8 \%$ of the total cases and $10 \%$ of total deaths worldwide. Over $70 \%$ of new cases and deaths occur in developing countries and GC is a leading cause of cancer-associated mortality in China (1,2). Dysregulation of normal signaling pathways is a critical process in the pathogenesis of GC $(3,4)$. Mitogen-activated protein kinase (MAPK) signaling activities play important roles in many of the processes involved in the initiation and genesis of cancer, and MAPK signaling pathway abnormalities have been shown to be involved in various human malignancies, including GC (5-7).

MAPK pathways constitute a highly conserved family of kinase modules $(8,9)$ that it serve to relay information from extracellular signals to the effectors which control various cell processes such as proliferation, differentiation, migration and apoptosis (10-13). MAPKs are activated by phosphorylation on the threonine and tyrosine residues of a conserved signature T-X-Y motif within the activation loop of the kinase (5). There are MAPK phosphatases (MKPs) that act as negative regulators of MAPK activity in mammalian $(14,15)$. The MKPs constitute a distinct subgroup of 10 catalytically active enzymes within the larger family of cysteine-dependent dual-specificity protein phosphatases (DUSPs) $(16,17)$. The dual-specificity phosphatase 9 (DUSP9) gene, also known as mitogen-activated protein kinase phosphatase 4 (MKP4), was first described in 1997 by Muda et al (18). This gene is a member of the large family of protein tyrosine phosphatases (PTPs) (18). It consists of 4 exons and has a size of $8,884 \mathrm{bp}$ and is localized on chromosome $\mathrm{Xq} 28$, which codes for a functional protein of $41.9 \mathrm{kDa}$.

DUSP9 has been shown to be downregulated in colorectal cancer, renal and hepatocellular carcinomas, and squamous 
Table I. PCR primer sequences for RT-qPCR and bisulfite sequencing PCR assay.

\begin{tabular}{|c|c|c|c|}
\hline Primer & Sequence & $\begin{array}{l}\text { Sequence product } \\
\text { size (bp) }\end{array}$ & $\begin{array}{c}\text { Annealing } \\
\text { temperature }\left({ }^{\circ} \mathrm{C}\right)\end{array}$ \\
\hline \multicolumn{4}{|l|}{ RT-qPCR } \\
\hline DUSP9 & $\begin{array}{l}\text { F: 5'-CAGCCGTTCTGTCACCGTC-3' } \\
\text { R: 5'-CAAGCTGCGCTCAAAGTCC-3' }\end{array}$ & 208 & 60 \\
\hline GAPDH & $\begin{array}{l}\text { F: 5'-GGAGCGAGATCCCTCCAAAAT-3' } \\
\text { R: 5'-GGCTGTTGTCATACTTCTCATGG-3' }\end{array}$ & 197 & 60 \\
\hline CCND1 & $\begin{array}{l}\text { F: 5'-GCTGCGAAGTGGAAACCATC-3' } \\
\text { R: 5'-CCTCCTTCTGCACACATTTGAA-3' }\end{array}$ & 135 & 60 \\
\hline CDK6 & $\begin{array}{l}\text { F: 5'-TCTTCATTCACACCGAGTAGTGC-3' } \\
\text { R: 5'-TGAGGTTAGAGCCATCTGGAAA-3' }\end{array}$ & 130 & 61 \\
\hline p21 & $\begin{array}{l}\text { F: 5'-TGTCCGTCAGAACCCATGC-3' } \\
\text { R: 5'-AAAGTCGAAGTTCCATCGCTC-3' }\end{array}$ & 139 & 60 \\
\hline \multicolumn{4}{|c|}{ Bisulfite sequencing PCR assay } \\
\hline BSP-DUSP9 & $\begin{array}{l}\text { F: 5'-AATAGAGGTTTGTAGGTGGGAG-3' } \\
\text { R: 5'-CCCTACCCAAAAAAAACACT-3' }\end{array}$ & 138 & 58 \\
\hline
\end{tabular}

DUSP9, dual-specificity phosphatase 9; BSP, bisulfite sequencing PCR; F, forward; R, reverse.

cell carcinoma (19-22). In a mouse model, the tumor-suppressor capacity of DUSP9 was confirmed on squamous cell carcinoma and NSCLC tissues (17). However, to the best of our knowledge, no studies have been performed regarding DUSP9 expression in human GC tissues. Additionally, no studies have been performed with the regard to the mechanism of DUSP9 inactivation in GC.

The present study was performed using 30 matched GC and normal gastric mucosa tissues. The analysis was conducted using bisulfite sequencing PCR (BSP) after bisulfite treatment of DNA. The lentiviral transfection of these cells with an inducible DUSP9 transfect led to a re-expression of DUSP9, was resulting in a repression of proliferation in GC cell lines.

\section{Materials and methods}

Ethics statement. For use of these clinical materials for the present study, written informed consent from all the patients and approval from the Ethics Committees of the Nanfang Hospital of Guangdong Province were obtained. The subjects involved in this study provided signed written informed consent. The study was approved by the Ethics Committee of Southern Medical University, Guangzhou, Guangdong, China. Animal experimentation was approved by the Ethics Committee of Animal Research at Southern Medical University. The experimental protocol was established according to the associated national guidelines from the Ministry of Science and Technology of China.

Cell culture and tissue collection. GC cell lines MKN-1, SCP26, SCP17, SCP51, M5, SW480, SW620, and LOVO were maintained in RPMI-1640 supplemented with $10 \%$ FCS (MP Biomedicals) at $37^{\circ} \mathrm{C}$ in a $5 \% \mathrm{CO}_{2}$ incubator. The GC and normal mucosa samples were collected from the Nanfang Hospital, the Affiliated Hospital of Southern
Medical University, Guangzhou, China. All the specimens had confirmed pathological diagnosis and were staged according to the 2009 UICC-TNM classification of malignant tumors.

$R N A$ isolation, reverse transcription, and $R T-q P C R$. Total RNA was extracted from frozen tumor samples using TRIzol reagent (Invitrogen, Carlsbad, CA, USA). cDNA was synthesized from $500 \mathrm{ng}$ total RNA using the PrimeScript RT Master Mix (Perfect Real Time, DRR036A; Takara, Tokyo, Japan). mRNA expression levels were quantified using quantitative PCR in a 96-well format by a SYBR-Green-based approach using a 7500 Fast Real-Time PCR system (Applied Biosystems) and SYBR ${ }^{\circledR}$ Premix Ex Taq ${ }^{\mathrm{TM}}$ II (Takara Bio, Inc., Ohtsu, Japan) in a final volume of $20 \mu 1$ including $100 \mathrm{ng}$ cDNA and $0.4 \mathrm{pmol} / \mu 1$ of each primer. The thermal cycling conditions included an initial denaturation for $30 \mathrm{sec}$ at $95^{\circ} \mathrm{C}$ and 40 cycles consisting of an annealing step at $95^{\circ} \mathrm{C}$ for $5 \mathrm{sec}$ and an extension step at $60^{\circ} \mathrm{C}$ for $20 \mathrm{sec}$. Each sample was analyzed in triplicate. The sequences primer used for PCR are shown in Table I. The relative expression of genes were calculated by the $2^{-\Delta \Delta \mathrm{Ct}}$ method. Data are presented as the relative quantity of target mRNA normalized to the expression of GAPDH mRNA and relative to a calibrator sample. Each assay was performed three times.

Bisulfite sequencing. Clinical samples were homogenized and digested overnight with proteinase $\mathrm{K}$. Clinical sample DNA was modified by the bisulfite reaction using an EpiTect Bisulfite kit (Qiagen, Germany) and quantified using a NanoDrop instrument (Thermo Scientific, Rockford, IL, USA). Modified DNA (40 ng/reaction) was amplified by PCR, using $0.2 \mu \mathrm{M}$ of each primer, 2 units of HotStart Taq DNA polymerase, and $0.2 \mathrm{mM}$ of each dNTP per reaction. Cycling programs (Applied Biosystems, Life technologies, USA) were $95^{\circ} \mathrm{C}$ for $5 \mathrm{~min}$, then 40 cycles of $95^{\circ} \mathrm{C}$ for $10 \mathrm{sec}, 60^{\circ} \mathrm{C}$ for $30 \mathrm{sec}$, and $72^{\circ} \mathrm{C}$ for $20 \mathrm{sec}$, 
Table II. shRNA sequences for DUSP9.

\begin{tabular}{|c|c|c|}
\hline & DUSP9 & Sequence \\
\hline \multirow[t]{2}{*}{1015} & Sense & 5'-AGGCCATTGAGTTCATTGATTCAAGAGATCAATGAACTCAATGGCCTTTTTTTACGCGT-3' \\
\hline & Antisense & 5'-ACGCGTAAAAAAAGGCCATTGAGTTCATTGATCTCTTGAATCAATGAACTCAATGGCCT-3' \\
\hline
\end{tabular}

DUSP9, dual-specificity phosphatase 9.

followed by a 5 -min incubation at $72^{\circ} \mathrm{C}$. PCR products were examined after gel electrophoresis in $1.5 \%$ agarose to confirm that a single band was obtained. Forward and reverse primer sequences of DUSP9 for bisulfite sequencing (Invitrogen) are shown in Table I, and together they amplified a 138-bp product containing $11 \mathrm{CpG}$ sites in the promoter. Sequence homologies were identified using the BLAST program of the National Center for Biotechnology Information available at http://www. ncbi.nlm.nih.gov/BLAST/.

Western blot analysis. Cell lysate was prepared using RIPA buffer $(50 \mathrm{mM}$ Tris $\mathrm{pH} 7.4,0.15 \mathrm{M} \mathrm{NaCl}, 1 \%$ Triton X-100, $1 \%$ sodium deoxycholate and $0.1 \%$ SDS with $1 \mathrm{mM}$ PMSF) with protease inhibitors and quantified using the BCA protein assay (BioTek, China). Protein $(20 \mu \mathrm{g})$ was loaded onto a $10 \%$ SDS-PAGE gel (Bio-Rad, Hercules, CA, USA) that was then transferred onto a PVDF membrane (Amersham Biosciences, Buckinghamshire, UK) and incubated with rabbit anti-DUSP9 (1:2,000 diluted, cat no. ab54941-100; Abcam, Cambridge, UK), rabbit anti-p21 (1:1,000 diluted; Cell Signaling Technology, Danvers, MA, USA), rabbit anti-CDK4 (1:2,000 diluted), rabbit anti-CDK6 (1:3,000 diluted), rabbit anti-CCND1 (1:2,000 diluted) and rabbit anti-c-Jun (1:1,000 diluted) (all from Santa Cruz Biotechnology, Inc., Santa Cruz, CA, USA) at $4^{\circ} \mathrm{C}$ overnight in blocking buffer (3\% non-fat dry milk/BSA in TBS) followed by incubation with HRP-conjugated secondary anti-mouse anti-body (1:5,000 diluted; ZSGB-Bio, China). The bands were visualized by an ECL Western Blot kit (CWBio Technology, Beijing, China). The images were captured using a ChemiDoc ${ }^{\mathrm{TM}}$ XRS+ Molecular Imager (Bio-Rad).

Tumor growth assay. Female BALB/c nude mice aged 4-5 weeks were purchased from the Central Animal Facility of Southern Medical University. Animal handling and experimental procedures were approved by the Animal Experimental Ethics Committee of CUHK. Following fluorescence-activated cell sorting, the green fluorescence protein-positive cells were isolated and a total of $5 \times 10^{5}$ infected cells were injected subcutaneously into the dorsal flank of nude mice. Each group contained 7 mice and the experiment was repeated three times.

Plasmid construction and transfection. Eukaryotic expression wild-type vectors (pEGFP-DUSP9) were constructed by Guangzhou Sagene (Sagene Incorporation, Guangzhou, China). MKN-1 cells were transfected with empty vector (pEGFP-C1), and the wild-type vector (pEGFP-DUSP9) using Lipofectamine 2000 (Invitrogen). Culture medium containing G418 (Sigma-Aldrich, St. Louis, MO, USA) was used to select stable transfectants.
Table III. siRNA sequences of DUSP9.

\begin{tabular}{lll}
\hline \multicolumn{2}{c}{ DUSP9 } & Sequence \\
\hline 921 & Sense & 5'-CUCCCAAACUUCUUCGAGAdTdT-3' \\
& Antisense & 3'-dTdTTCUCGAAGAAGUUUGGGAG-5'
\end{tabular}

DUSP9, dual-specificity phosphatase 9.

Establishment of lentivirus-delivered LV-sh-DUSP9 and $L V$-DUSP9 in GC cells. The preparation of lentivirus-expressing human DUSP9 short hairpin RNA (shRNA-1015, Table II) was performed using the pLVTHM-GFP lentiviral RNAi expression system. The lentiviral particles were used to infect MKN-1 GC cell lines. Lentivirus (GV208, Ubi-MCS-EGFP) particles carrying DUSP9 and its control were purchased from GeneChem (Shanghai, China). The lentiviral transduction of MKN-1 cells was carried out according to the manufacturer's instructions. The resulting cells were seeded in 96-well plates and cultured for 3 weeks to produce a stable DUSP9-overexpressing MKN-1 cells and MKN-1 control cells. The high expression of DUSP9 was validated by RT-qPCR.

Transient transfection with siRNAs for DUSP9. siRNAs were transfected at a working concentration of $100 \mathrm{nmol} / \mathrm{l}$ using Lipofectamine 2000 reagent (Invitrogen). The siRNA for DUSP9, a non-specific control were all purchased from Guangzhou RiboBio Co., Ltd. (Guangzhou, China). The sequence of each gene and their controls are shown in Table III. Twenty-four hours before transfection, MKN-1 cells were plated onto a 96 -well plate or a 6 -well plate at a $30-50 \%$ confluence. GC cells were then transfected into MKN-1 cells using TurboFect siRNA transfection reagent (Fermentas, Vilnius, Lithuania) according to the manufacturer's instructions. The cells were collected after 48-72 h for subsequent experiments.

Colony formation assay. After $72 \mathrm{~h}$ of infection, the cells were plated in 6-well plates at a concentration of 200 cells/well and grown for 2 weeks. After 2 weeks, the cells were washed twice with PBS, fixed with methanol/acetic acid (3:1, v/v), and stained with $0.5 \%$ crystal violet. The number of colonies was counted under the microscope (Olympus FV1000; Olympus, Tokyo, Japan).

Cell proliferation and cell-cycle analyses. Cell proliferation was analyzed using an MTT assay (Sigma-Aldrich). Briefly, 
A

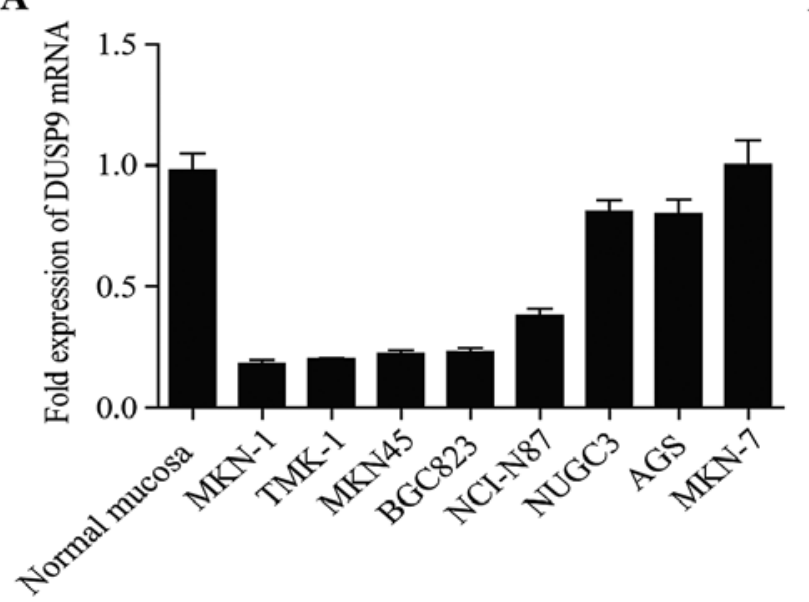

B

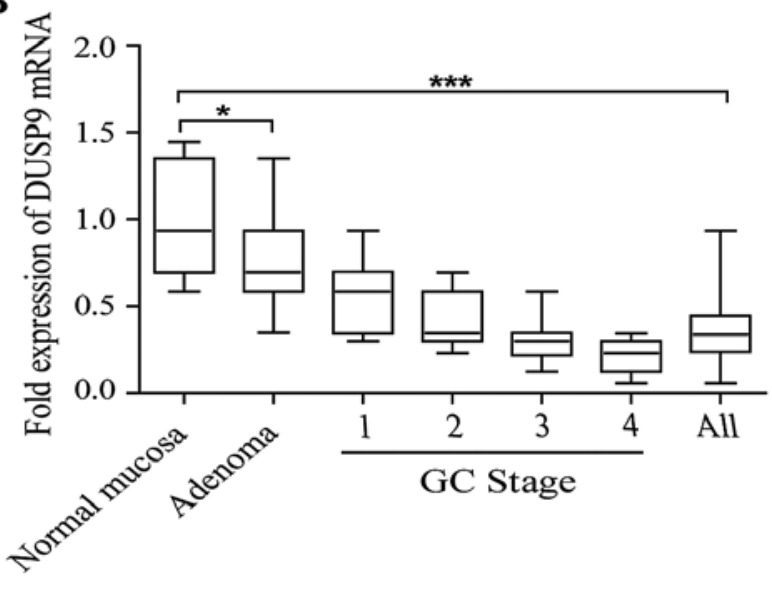

Figure 1. The expression of DUSP9 was reduced in GC cell lines and clinical specimens. (A) Expression of DUSP9 in 8 GC cell lines. (B) Average expression level of DUSP9 in human GC specimens (17, stage 1; 36, stage 2; 43, stage 3 and 28, stage 4; for a combined total of 124 GC specimens) and 24 normal gastric mucosa and 30 gastric adenoma tissues. ${ }^{*} \mathrm{P}<0.05,{ }^{* * *} \mathrm{P}<0.0001$. RNA abundance was normalized to GAPDH RNA. DUSP9, dual-specificity phosphatase 9 ; GC, gastric cancer.

MKN-1 cells $\left(5 \times 10^{3}\right)$ were plated onto 96-well plates, respectively, in $100 \mu \mathrm{l}$ of growth medium and allowed to adhere overnight. The cells were then transfected with DUSP or Si-DUSP9 and control, respectively. At different time-points (24, 48, and $72 \mathrm{~h})$, the culture medium was removed and replaced with culture medium containing $10 \mu \mathrm{l}$ of sterile MTT dye $(5 \mathrm{mg} / \mathrm{ml})$. After incubation at $37^{\circ} \mathrm{C}$ for $4 \mathrm{~h}$, the MTT solution was removed, and $150 \mu \mathrm{l}$ dimethyl sulfoxide (DMSO) was added to dissolve the formazan crystals. Spectrometric absorbance at $490 \mathrm{~nm}$ was measured by a BioTek ELx800 microplate photometer (SN211805; BioTek Instruments, Winooski, VT, USA).

For the cell-cycle analysis, LV-DUSP9-infected MKN-1 cells, LV-sh-DUSP9-infected MKN-1 cells and negative control cells were fixed in 70\% ice-cold ethanol for $48 \mathrm{~h}$ at $4^{\circ} \mathrm{C}$, stained by incubation with PBS containing $10 \mu \mathrm{g} /$ $\mathrm{ml}$ propidium iodide and $0.5 \mathrm{mg} / \mathrm{ml} \mathrm{RNase}$ A for $15 \mathrm{~min}$ at $37^{\circ} \mathrm{C}$, and analyzed for the DNA content of labeled cells by a FACSCalibur cytometer. Each experiment was performed in triplicate.

Statistical analysis. Data are presented as mean \pm SD. SPSS 13.0 software (SPSS Inc., Chicago, IL, USA) and GraphPad software (GraphPad Software, Inc., La Jolla, CA, USA) were used to analyze the data for statistical significance. A two-tailed Student's t-test was used for comparisons of two independent groups. One-way ANOVA was used to determine the differences between groups for all in vitro analyses and the log-rank Mantel-Cox test for in vitro and in vivo analyses. $\mathrm{P}<0.05$ was considered to be statistically significant.

\section{Results}

DUSP9 expression is reduced in GC cell lines. A panel of human GC cell lines was first analyzed to quantify the expression level of DUSP9. The results showed that the expression level of DUSP9 was decreased in the seven GC cell lines examined, compared with the normal gastric mucosa tissues (Fig. 1A). DUSP9 transcript levels were significantly decreased in all the stages of GC compared with the normal gastric mucosa tissues (Fig. 1B). DUSP9 transcript levels were also reduced in premalignant lesions (adenomas) compared with those in normal gastric mucosa tissues, indicating that DUSP9 loss occurs early in the progression to tumorigenesis. These data supported that the DUSP9 transcript level was downregulated in GC.

DUSP9 is silenced via hypermethylation in malignancy. The DUSP9 promoter contains a large CpG island from -1297 to +1104 from the transcription start site (TSS, Fig. 2A) based on the criteria and algorithm described by Li and Dahiya (23). We performed BSP in 30 matched GC and normal gastric mucosa tissues. BSP of the DUSP9 promoter included $11 \mathrm{CpG}$ sites (Fig. 2B, left panel). In normal gastric mucosa tissues, the DUSP9 promoter demonstrated low methylation levels (average methylation level was 20.3\%). By contrast, DUSP9 promoter methylation was significantly increased at each individual $\mathrm{CpG}$ site examined and reached an average of $74.5 \%$ in the cancerous tissues (Fig. 2B, right panel, $\mathrm{P}<0.001$ ).

To confirm the role of DNA methylation in the transcriptional regulation of DUSP9, we treated MKN-1 cells with 2 or $8 \mu \mathrm{M} \mathrm{5-aza-2}$-deoxycytidine for $72 \mathrm{~h}$ and examined DUSP9 promoter methylation and mRNA expression changes. DUSP9 gene expression was restored following 5-aza-2'-deoxycytidine treatment (Fig. 2D and E). This re-expression was accompanied by a decrease in promoter DNA methylation from 94 to $72 \%$ (Fig. 2C). These results indicated that promoter hypermethylation is one mechanism mediating transcriptional silencing of DUSP9 in GC.

DUSP9 induces growth inhibition in GC cells in vitro and in vivo. To examine the effect of DUSP9 on cell growth, MKN-1 cells were transiently transfected with DUSP9 vector or negative control vector, respectively. As shown in Fig. 3A, the results of MTT assay showed that DUSP9 inhibited cell growth in MKN-1 cells by $52 \%(\mathrm{P}<0.01)$, whereas Si-DUSP9 


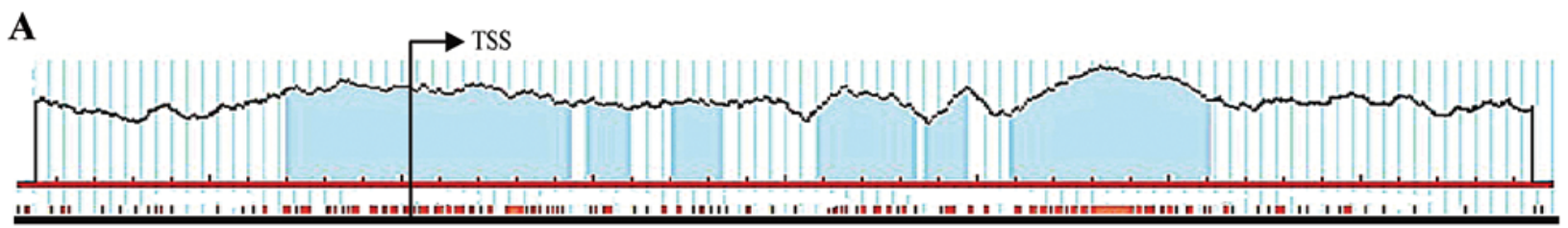

Bisulfite sequencing PCR assay
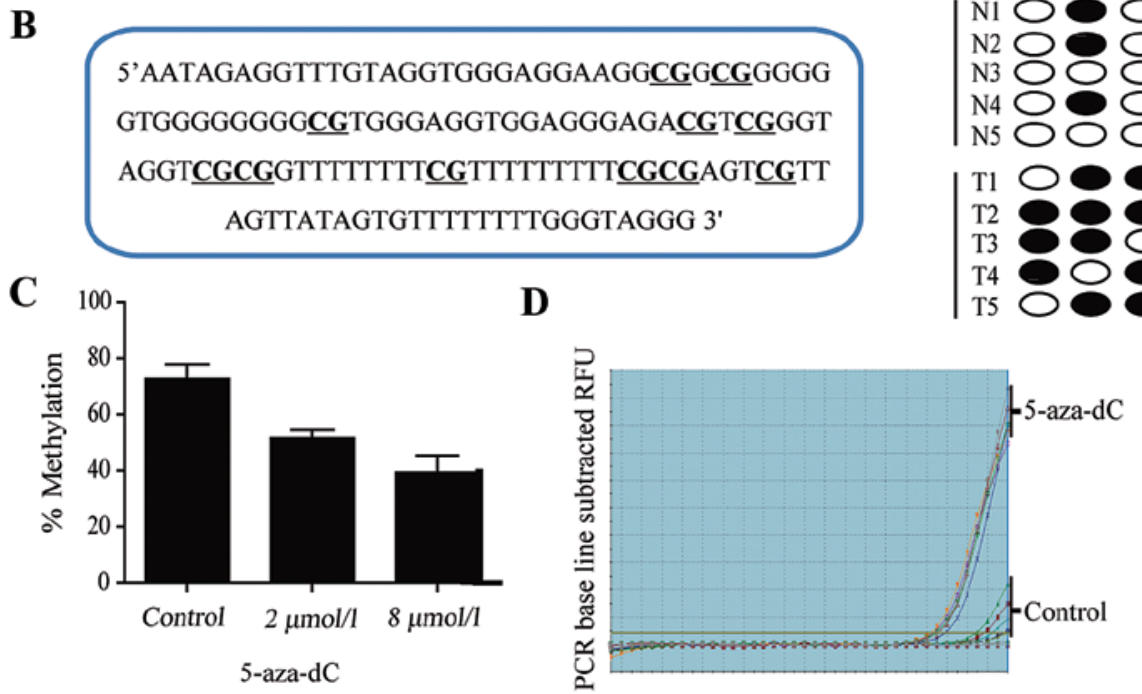

DUSP9 promoter methylation status

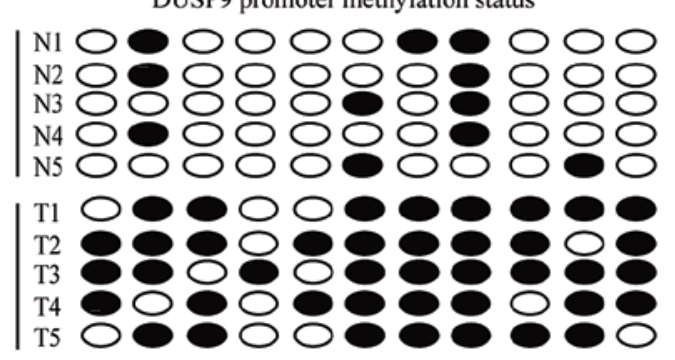

$\mathbf{E}$

5-aza-dC $\quad 0 \mu \mathrm{mol} / 1 \quad 2 \mu \mathrm{mol} / 1 \quad 8 \mu \mathrm{mol} / 1$

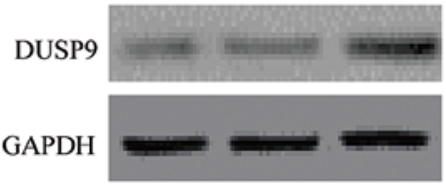

Figure 2. DUSP9 expression is silenced via promoter hypermethylation in GC. (A) Schematic representation of the human DUSP9 promoter. The CpG island of DUSP9 extends from -1297 to +1104 from TSS. Each red tick mark represents is one CpG site. The arrows indicate the TSS. To determine the methylation level, BSP was carried out on $11 \mathrm{CpG}$ sites extending from -215 to -78 from TSS (underlined). (B) Bisulfite sequencing evaluation of CpG island methylation of the $11 \mathrm{CpG}$ sites of DUSP9 promoter in $\mathrm{GC}(\mathrm{T}=30)$ and normal mucosa tissues $(\mathrm{N}=30)$. Left panel, location of $11 \mathrm{CpG}$ sites in the DUSP9 promoter. Right panel, open circles, unmethylated $\mathrm{CpG}$; closed circles, methylated CpG. (C) BSP in MKN-1 cells showed detection of DUSP9 methylation status following treatment with 5-aza-dC for $72 \mathrm{~h}$. To determine the methylation level (\%), BSP was carried out on $11 \mathrm{CpG}$ sites. (D) RT-qPCR in MKN-1 cells showed detection of DUSP9 mRNA following treatment with vehicle or 5-aza-dC for $72 \mathrm{~h}$. (E) Treatment with 5-aza-dC restoring DUSP9 expression in MKN-1. 5-aza-dC treatment can lead to DNA demethylation via inhibition of DNA methyltransferase activity. Data are presented as the average of triplicate measurements from duplicate experiments. TSS, transcriptional start site; 5-aza-dC, 5-aza-2'-deoxycytidine; DUSP9, dual-specificity phosphatase 9; GC, gastric cancer; BSP, bisulfite sequencing PCR.

promoted cell growth in MKN-1 cells by $74 \%(\mathrm{P}<0.01)$. By contrast, the DUSP9-negative control or Si-DUSP9 control had no effect on cell growth, indicating that the effect caused by DUSP9 was highly specific. As demonstrated by the colony formation assay, DUSP9-infected MKN-1 cells exhibited much fewer and smaller colonies compared with LV-coninfected cells (Fig. 3B, $\mathrm{P}<0.01$ ).

We used lentiviral vectors to stably restore the expression of DUSP9 in MKN-1 cells and examined cell-cycle distribution. Compared with the negative control, LV-DUSP9-infected MKN-1 cells showed an increased percentage of cells in the G1 phase and fewer cells in the $\mathrm{S}$ phase (Fig. $3 \mathrm{C}, \mathrm{P}<0.01$ ), while the cell-cycle distribution had a significant difference between LV-sh-DUSP9 control and LV-sh-DUSP9-transfected cells (Fig. 3C, $\mathrm{P}<0.0001$ ). These results suggested that the growth-suppressive effect of DUSP9 was partly due to a G1 phase arrest.

MKN-1 cells were infected with LV-sh-DUSP9 and then injected subcutaneously into the dorsal flank of nude mice. As early as 2 weeks post-implantation, the growth of transplanted tumors between two groups became statistically significant. At 25 days after implantation, those mice injected with LV-sh-DUSP9 carried larger burdens. As compared with the LV-sh-DUSP9-treated group, the average tumor volume of the control group was markedly reduced by $>60 \%$ (Fig. 3D and E, $\mathrm{P}<0.001$ ). The loss of animals in both arms of the study is visible in Fig. 3F, where the survival curve for the control arm decreased considerably after week 4, with the difference between the two groups approaching significance, as determined by the log-rank Mantel-Cox test $(\mathrm{P}<0.01)$.

DUSP9 inhibits progression of the cell cycle at S-G2/M phase by regulating cell cycle-related molecules. To investigate the mechanisms leading to loss of cell proliferation by DUSP9, we assessed whether the observed inhibitory effects of DUSP9 on cell proliferation were due to induction of cell-cycle arrest. As previously described, infection with LV-sh-DUSP9 increased the percentage of cells in the $S$ and $G 2 / M$ phase. DUSP-mediated cycle-related molecules downregulation was observed in the transient expression system in MKN-1 cells (Fig. 4A). RT-qPCR showed that, the mRNA expression level of the CCND1 gene was shown to be decreased 0.81-fold at $24 \mathrm{~h}$ and 0.35 -fold at $48 \mathrm{~h}$, and this level was sustained up to $72 \mathrm{~h}$ after the transfection of DUSP9 into MKN-1 cells. The mRNA expression levels of CDK4 and CDK6 in DUSP9 gene-transfected MKN-1 cells were at the same level as that of CCND1 mRNA expression throughout the time course. By contrast, Si-DUSP9-mediated cycle-related molecules 
A

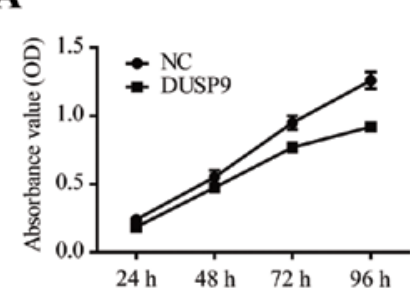

C
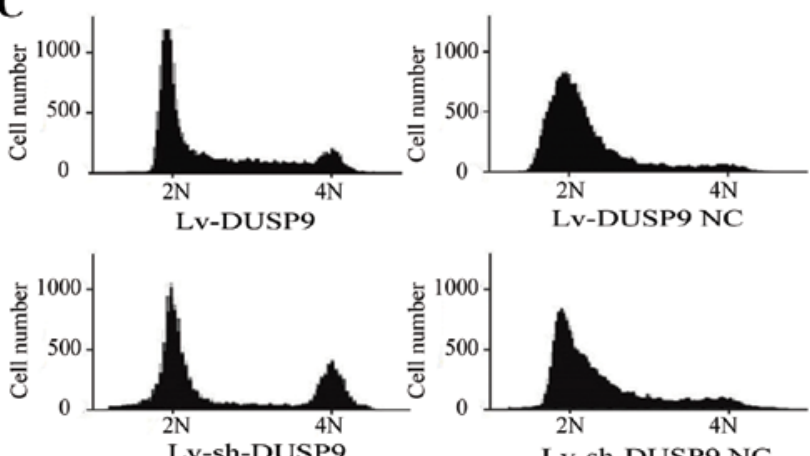

D

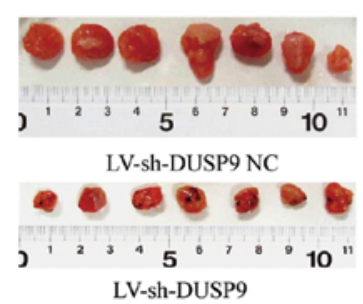

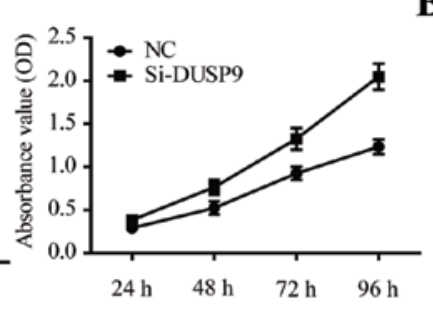

B

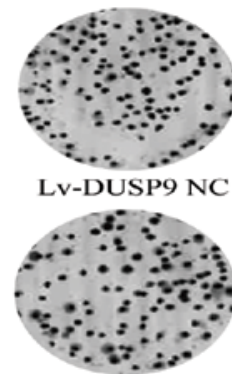

Lv-sh-DUSP9 NC

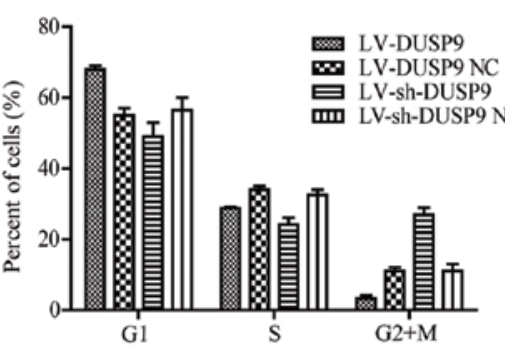

F
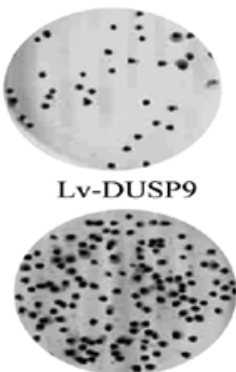

v-sh-DUSP9

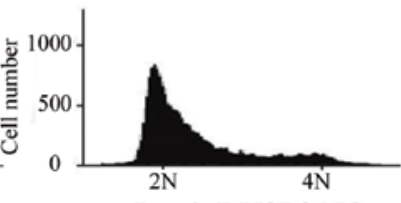

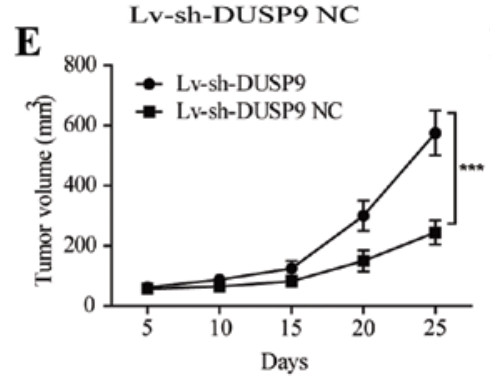

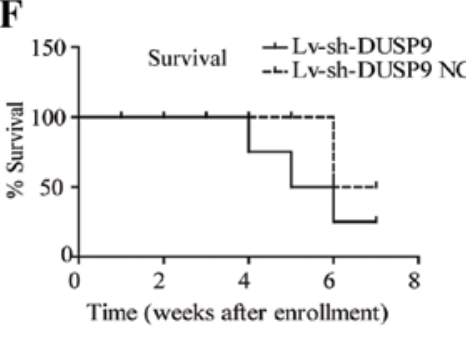

Figure 3. Enforced expression of DUSP9 induces growth inhibition in GC cells. (A) Effect of DUSP9/Si-DUSP9 on cell proliferation was measured by MTT assay following transfection with DUSP9/Si-DUSP9 in MKN-1 cells. (B) Representative images of colony formation assay of LV-DUSP9-infected/LV-shDUSP9-infected MKN-1 cells. (C) Representative histograms for cell-cycle distribution of LV-DUSP9-infected/LV-sh-DUSP9-infected MKN-1 cells. (D) MKN-1 cells were infected with LV-sh-DUSP9 and injected subcutaneously into nude mice. Twenty-five days after implantation, LV-sh-DUSP9-infected cells produced bigger tumors than the control cells. (E) Growth curve of tumor volumes. Each data point presented the mean \pm SD of 7 mice. (F) Survival in nude mice in the indicated groups. DUSP9, dual-specificity phosphatase 9; GC, gastric cancer.

A

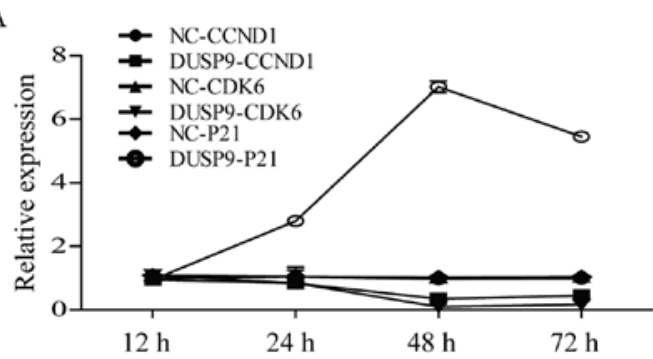

$\mathrm{C}$

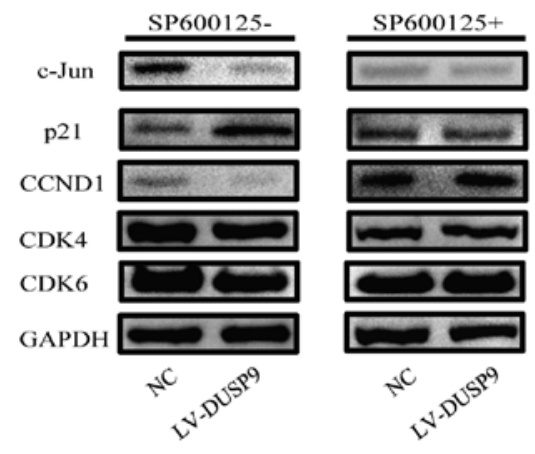

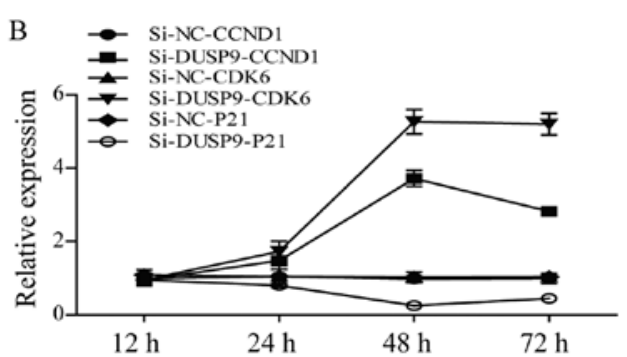

D

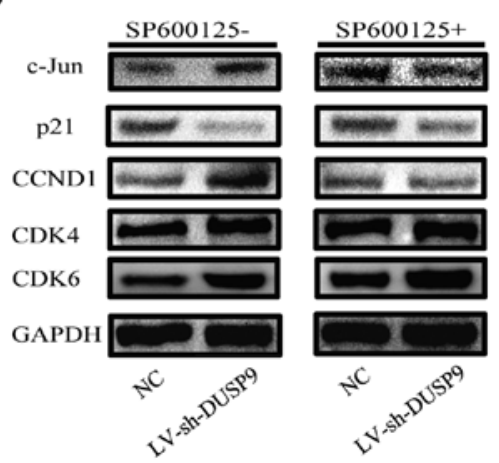

Figure 4. DUSP9 is a negative regulator of cell cycle-related molecules activity in GC cells. (A and B) Quantitative PCR analysis of CCND1, CDK6, and p21 in MKN-1 cells, induced by the transient transfection of the DUSP/Si-DUSP9 gene. (C and D) Western blot analyses of c-Jun, CCND1, CDK4, CDK6 and p21 in the indicated cell groups. DUSP9, dual-specificity phosphatase 9; GC, gastric cancer. 
upregulation was observed in the transient expression system in MKN-1 cells (Fig. 4B).

We also examined the effect of DUSP9 on cell cycle-regulatory molecules, including c-Jun, CCND1, CDK4, CDK6, and p21 (Fig. 4C, left). The infection of MKN-1 cells with LV-DUSP9 resulted in downregulation in the levels of c-Jun protein as well as the levels of CCND1. Downregulation of CDK4 and CDK6 was also observed in MKN-1 cells. The level of p21 protein increased markedly following the infection of MKN-1 cells with LV-DUSP9. By contrast, the infection of MKN-1 cells with LV-sh-DUSP9 resulted in upregulation in the levels of c-Jun protein as well as the levels of CCND1. Upregulation of CDK4 and CDK6 was also observed in MKN-1 cells. In addition, the expression of p21 increased in LV-sh-DUSP9-infected cells in comparison with the controls. However, the infection of MKN-1 cells with LV-sh-DUSP9/LV-DUSP9 resulting in regulation of the levels of cycle-related molecules was inhibited when the JNK inhibitor SP600125 was added (Fig. 4C, right and 4D, right), suggesting that the drug inhibits the DUSP9-mediated JNK activation of c-Jun, CCND1, CDK4, CDK6 and inactivation of $\mathrm{p} 21$. Thus, the results of the present study confirm that DUSP9 induces regulation in the levels of cycle-related molecules via JNK signaling.

\section{Discussion}

DNA methylation of promoter-associated $\mathrm{CpG}$ islands may function as an alternate mechanism of silencing tumor-suppressor genes in numerous neoplasias, including GC. De novo methylation of genes appears to be an early and frequent event in most neoplasias (24-28). Hypermethylation occurs at different stages in the development of cancer and in different cell networks (29). In the genesis of many types of cancer, hypermethylation of the $\mathrm{CpG}$ islands in the promoter regions of tumor-suppressor genes is a major event, and is able to affect genes involved in the cell cycle, DNA repair, the metabolism of carcinogens, cell-to-cell interaction, apoptosis, and angiogenesis $(30,31)$. In the present study, the expression of DUSP9 has been reported to be reduced in different types of cancer (19-22). The results show that, DUSP9 was frequently methylated in human GC and the expression of DUSP9 was suppressed by promoter region methylation.

The aim of this study was to examine the $\mathrm{CpG}$ island methylation status of DUSP9, on 30 clinical GC samples and selected corresponding tumor-free tissues, using BSP for the determination of the promoter methylation status. The methylation status of the tumor samples was compared to that of the corresponding tumor-free samples. To the best of our knowledge, this is the first study using BSP for a DUSP9 methylation analysis on clinical GC samples. The determination of the methylation status for each sample and $\mathrm{CpG}$ island was successful.

DUSP9 was silenced by promoter region hypermethylation and G2/M phase arrest was induced by DUSP9 in MKN-1 cells. DUSP9 inhibits GC growth, suggesting that DUSP9 is a tumor suppressor in human GC. The expression of CCND1, c-Jun, CDK4, CDK6 was upregulated whereas p21 was downregulated by DUSP9 in MKN-1 cells. However, the infection of MKN-1 cells with LV-sh-DUSP9/LV-DUSP9 resulted in the regulation in the levels of cycle-related molecules being suppressed when the JNK inhibitor SP600125 was added. Our results suggest that cell proliferation was suppressed by DUSP9 by inhibiting c-Jun, which was induced by JNK signaling. Loss of DUSP9 with progression of GC allows the growth-promoting activities of JNK to persist, while the invasive and growth inhibitory effects are lost. The increased levels of DUSP9 expression observed in human GC cell lines were inversely correlated with JNK activity and markers of apoptosis, indicating that DUSP9 may be anti-proliferative in GC via its activity towards JNK.

In conclusion, DUSP9 was frequently methylated in human GC and the expression of DUSP9 was silenced by the promoter region hypermethylation. DUSP9 suppresses GC proliferation by inhibiting JNK signaling pathways. As was the case in the present study, overexpression of DUSP9 correlated with reduced JNK activity. However, DUSP9induced JNK kinase inactivation can be specifically blocked by the inhibitor SP600125. This results suggest that therapeutic intervention to increase the expression or activity of DUSP9 may enable the activation of the anti-proliferative signals in malignant cells.

\section{Acknowledgements}

This study was supported by the Medicine and Health grant from Wenzhou Bureau of Science and Technology (grant no. Y20140281). The authors thank Ming Li (Sun Yat-Sen University Cancer Center, Guangzhou, People's Republic of China) and Liang Wang (Zhejiang University, Hangzhou, Zhejiang, People's Republic of China) for technical support.

\section{References}

1. Jemal A, Bray F, Center MM, Ferlay J, Ward E and Forman D: Global cancer statistics. CA Cancer J Clin 61: 69-90, 2011.

2. Hartgrink HH, Jansen EP, van Grieken NC and van de Velde CJ: Gastric cancer. Lancet 374: 477-490, 2009.

3. Wu WK, Cho CH, Lee CW, Fan D, Wu K, Yu J and Sung JJ: Dysregulation of cellular signaling in gastric cancer. Cancer Lett 295: 144-153, 2010.

4. Venerito M, Nardone G, Selgrad M, Rokkas T and Malfertheiner P: Gastric cancer - epidemiologic and clinical aspects. Helicobacter 19 (Suppl 1): 32-37, 2014

5. Wagner EF and Nebreda AR: Signal integration by JNK and p38 MAPK pathways in cancer development. Nat Rev Cancer 9: 537-549, 2009.

6. Dong HW, Zhang S, Sun WG, Liu Q, Ibla JC, Soriano SG, Han XH, Liu LX, Li MS and Liu JR: $\beta$-ionone arrests cell cycle of gastric carcinoma cancer cells by a MAPK pathway. Arch Toxicol 87: 1797-1808, 2013.

7. Chen P, Zhao D, Sun Y, Huang L, Zhang S and Yuan Y: Protein inhibitor of activated STAT-1 is downregulated in gastric cancer tissue and involved in cell metastasis. Oncol Rep 28: 2149-2155, 2012.

8. Chen L, Hu W, Tan S, Wang M, Ma Z, Zhou S, Deng X, Zhang Y, Huang C, Yang G, et al: Genome-wide identification and analysis of MAPK and MAPKK gene families in Brachypodium distachyon. PLoS One 7: e46744, 2012.

9. Berlier JL, Rigutto S, Dalla Valle A, Lechanteur J, Soyfoo MS, Gangji V and Rasschaert J: Adenosine triphosphate prevents serum deprivation-induced apoptosis in human mesenchymal stem cells via activation of the MAPK signaling pathways. Stem Cells 33: 211-218, 2015.

10. Dhillon AS, Hagan S, Rath O and Kolch W: MAP kinase signalling pathways in cancer. Oncogene 26: 3279-3290, 2007.

11. Chen Z, Cai Y, Zhang W, Liu X and Liu S: Astragaloside IV inhibits platelet-derived growth factor-BB-stimulated proliferation and migration of vascular smooth muscle cells via the inhibition of p38 MAPK signaling. Exp Ther Med 8: 1253-1258, 2014. 
12. Bernardo-Faura M, Massen S, Falk CS, Brady NR and Eils R Data-derived modeling characterizes plasticity of MAPK signaling in melanoma. PLoS Comput Biol 10: e1003795, 2014.

13. Park GB, Choi Y, Kim YS, Lee HK, Kim D and Hur DY: ROS-mediated JNK/p38-MAPK activation regulates Bax translocation in sorafenib-induced apoptosis of EBV-transformed B cells. Int J Oncol 44: 977-985, 2014.

14. Favata MF, Horiuchi KY, Manos EJ, Daulerio AJ, Stradley DA Feeser WS, Van Dyk DE, Pitts WJ, Earl RA, Hobbs F, et al: Identification of a novel inhibitor of mitogen-activated protein kinase kinase. J Biol Chem 273: 18623-18632, 1998.

15. Caunt CJ and Keyse SM: Dual-specificity MAP kinase phosphatases (MKPs): shaping the outcome of MAP kinase signalling. FEBS J 280: 489-504, 2013.

16. Wu GS: Role of mitogen-activated protein kinase phosphatases (MKPs) in cancer. Cancer Metastasis Rev 26: 579-585, 2007.

17. Keyse SM: Dual-specificity MAP kinase phosphatases (MKPs) and cancer. Cancer Metastasis Rev 27: 253-261, 2008.

18. Muda M, Boschert U, Smith A, Antonsson B, Gillieron C, Chabert C, Camps M, Martinou I, Ashworth A and Arkinstall S: Molecular cloning and functional characterization of a novel mitogen-activated protein kinase phosphatase, MKP-4. J Biol Chem 272: 5141-5151, 1997.

19. Liu Y, Lagowski J, Sundholm A, Sundberg A and KuleszMartin M: Microtubule disruption and tumor suppression by mitogen-activated protein kinase phosphatase 4. Cancer Res 67: 10711-10719, 2007.

20. Wu S, Wang Y, Sun L, Zhang Z, Jiang Z, Qin Z, Han H, Liu Z, Li X, Tang A, et al: Decreased expression of dual-specificity phosphatase 9 is associated with poor prognosis in clear cell renal cell carcinoma. BMC Cancer 11: 413, 2011.

21. Liu J, Ni W, Xiao M, Jiang F and Ni R: Decreased expression and prognostic role of mitogen-activated protein kinase phosphatase 4 in hepatocellular carcinoma. J Gastrointest Surg 17: 756-765, 2013
22. Jenner S, Wiedorn KH and Techel D: Development of a DUSP9 methylation screening assay. Pathol Oncol Res 21: 123-130, 2015.

23. Li LC and Dahiya R: MethPrimer: designing primers for methylation PCRs. Bioinformatics 18: 1427-1431, 2002.

24. Baylin SB, Herman JG, Graff JR, Vertino PM and Issa JP: Alterations in DNA methylation: a fundamental aspect of neoplasia. Adv Cancer Res 72: 141-196, 1998.

25. Baylin SB and Herman JG: DNA hypermethylation in tumorigenesis: epigenetics joins genetics. Trends Genet 16: 168-174, 2000.

26. Palau A, Perucho M, Esteller $M$ and Buschbeck M: First Barcelona conference on epigenetics and cancer. Epigenetics 9: 468-475, 2014.

27. Liu G, Liu YJ, Lian WJ, Zhao ZW, Yi T and Zhou HY: Reduced BMP6 expression by DNA methylation contributes to EMT and drug resistance in breast cancer cells. Oncol Rep 32: 581-588, 2014.

28. Minning C, Mokhtar NM, Abdullah N, Muhammad R,Emran NA, Ali SA, Harun R and Jamal R: Exploring breast carcinogenesis through integrative genomics and epigenomics analyses. Int $\mathrm{J}$ Oncol 45: 1959-1968, 2014.

29. Vizoso $M$ and Esteller M: German-Catalan workshop on epigenetics and cancer. Epigenetics 8: 998-1003, 2013.

30. Esteller M: Epigenetics in cancer. N Engl J Med 358: 1148-1159, 2008.

31. Guerrero-Preston R, Hadar T, Ostrow KL, Soudry E, Echenique M, Ili-Gangas C, Pérez G, Perez J, Brebi-Mieville P, Deschamps J, et al: Differential promoter methylation of kinesin family member la in plasma is associated with breast cancer and DNA repair capacity. Oncol Rep 32: 505-512, 2014. 